\title{
Improved Outcomes in Gallbladder Cancer with Early Diagnosis and Negative Nodal Status
}

\author{
Lauren Smithson, Christopher Keto, Lorenzo Ferguson, Sumet Silapaswan, Michael J. Jacobs, \\ Ramachandra Kolachalam, Jeffrey C. Flynn, Vijay K. Mittal
}

Department of Surgery, Providence Hospital and Medical Centers, Southfield, USA.

Email: vijay.mittal@providence-stjohnhealth.org

Received December $12^{\text {th }}, 2011$; revised January $15^{\text {th }}$, 2012; accepted February $10^{\text {th }}, 2012$

\begin{abstract}
Background: Gallbladder cancer (GBC) is a rare disease of the hepatobiliary tract characterized by silent presentation, poor prognosis, and limited therapy. Current imaging modalities, clinical symptoms and laboratory values are of limited value in diagnosis and tumour markers are used as a clinical adjunct. Five year survival is $5 \%-12 \%$ and a majority of patients survive less than 1 year. Early identification, negative nodal status, and extended cholecystectomy improve survival; adjuvant therapy does not appear to play a role. Objective: To evaluate the effects of stage and nodal status of GBC on survival by analyzing clinical and radiological factors leading to preoperative diagnosis and appropriate subsequent management. Results: Forty-three patients (31 female, 12 male) had primary GBC. Average age was 69 years. The most common presenting symptom was pain (65\%). Fifteen (35\%) patients presented with acute cholecystitis; 9 were suspicious for a mass. Ultrasound and computed tomography were the imaging modalities most often used. Preoperative diagnoses were made in only $7(16 \%)$ patients, whereas 16 (37\%) patients were diagnosed intra-operatively and 20 (47\%) post-operatively. Adjuvant chemotherapy was offered in 16 (37\%) patients. Average survival was 28 months (range 0.5 - 238 months), with a 5-year survival of $13 \%$ across all stages. Early GBC (stages 1 - 2) had a 5-year survival of 43\%, and late GBC (stages 3 - 4) had no survival at 5 years, with an average survival of 9 months. Positive nodes decreased survival. Conclusion: Our results mirror what has been reported in the literature. Most patients presented with acute cholecystitis, with confounding clinical data, but few were preoperatively diagnosed with GBC. Imaging was rarely diagnostic. Late stage GBC, as well as node-positive status, had dismal outcomes with low 1-year and 5-year survival rates. Improved preoperative suspicion of early GBC would allow for more intentional curative resections, before nodes become positive.
\end{abstract}

Keywords: Gallbladder; Carcinoma; Node Status; Survival

\section{Introduction}

Gallbladder cancer (GBC) is a rare disease of the hepatobiliary tract characterized by silent presentation, poor prognosis, and limited therapy. It is the most common malignancy of the biliary tract and the seventh most common gastrointestinal tract cancer, more so than cholangiocarcinoma [1]. It is found in $1 \%$ of all cholecystectomy specimens; twenty percent of GBC is diagnosed at the time of cholecystectomy for biliary colic or gallstones [2]. Although the pathological mechanism of GBC development is not understood, there is a known correlation with the presence of gallstones, possibly due to chronic inflammation. One to three percent of patients with cholelithiasis develop GBC over a 10 - 25 year progression $[3,4]$. Other gallstone-related factors are also associated with increased risk of GBC; women are 2 - 3 times more at risk than men, and incidence increases with age and obesity [3].

GBC lacks specific symptoms and there is usually a low clinical suspicion for malignancy [3]. Diagnostic imaging has been problematic in the determination of GBC. Computed tomography (CT) and ultrasound (US) are the main modalities used in the diagnosis of GBC, but results are limited, often mistaking GBC for more benign conditions like chronic cholelithiasis [3,5]. Other forms of imaging modalities, such as endoscopic ultrasound and magnetic resonance imaging, are confined to predicting the degree of invasion and resectability once GBC is diagnosed [6]. Post-operative scarring and inflammation, however, can skew imaging results, especially with studies such as positron emission tomography (PET) and CT scanning [2]. Prognosis is based on the depth of mucosal invasion and distal spread [6]. The paths of spread are lymphatic, vascular, neural, intrap- 
eritoneal, intraductal and by direct extension into the liver or other adjacent organs [7]. Nodal status plays a vital role in staging and in survival; paraaortic nodes, if positive, limit survival as much as do distant metastases [8]. Distant metastases include lungs, pleura, diaphragm and bone [2]. Staging is currently outlined by the American Joint Committee on Cancer (AJCC) staging system by tumor, node, and metastasis (TNM) [3].

Due to the nature of GBC, aggressive management is needed to improve survival. R0 resection is the goal for curative treatment, and a simple cholecystectomy can be curative if the tumor is restricted to the muscularis propria, but not into the serosa [7]. In advanced cancers, radical approaches are necessary and various procedures have been described for extended resections [3]. Regardless of the decision to surgically resect, preoperative planning is rare because of the incidental nature of early disease.

Adjuvant therapy does not have a confirmed role in the treatment of GBC to date. Survival is limited, with 5-year survival quoted as low as $60 \%$ for stage $0,39 \%$ for stage I, $15 \%$ for stage II, $5 \%$ for stage III, and $1 \%$ for stage IV [3]. The objectives of this study were to determine the demographics, nodal status, surgical treatments and survival statistics of patients with GBC over 20 years. Emphasis was placed on the survival statistics of patients with nodal status and advanced disease, as well as the imaging and clinicopathologic data that identified these cases, in order to formulate a better preoperative approach.

\section{Methods}

Clinicopathological data from 49 patients who underwent surgical resection of the gallbladder between 1989 and 2009 were analyzed. Of these, 43 patients had primary GBC and 6 patients had cancer metastatic to the gallbladder. Initially, a list of all pathological reports containing mention of cancer in the gallbladder was reviewed to determine the series that were specifically carcinoma gallbladder. A retrospective review of charts and pathology reports was undertaken, comparing the pre-, intra-, and post-operative diagnoses. Available radiology reports were reviewed and assessed qualitatively for suspicion of GBC or gallbladder masses. Data on palliative and adjuvant therapy were correlated through the tumor registry affiliated with the hospital site. Statistical analysis of demographics and risk factors was performed. Clinicopathologic correlates of clinical presentation and laboratory values, including tumor markers, were determined. Frequency and survival statistics, both 1 - and 5-year survival, were determined for patients with early and late GBC as well as for patients with single and multiple node-positive status. The study was concluded in
June 2009, where patients were either confirmed living, lost to follow up, or found deceased via public death record or personal physician record.

\section{Results}

Forty-nine patients were found to have GBC; 43 were primary, with 6 of those having multiple primaries including GBC. Six patients had metastases to the gallbladder from primary tumors of nasal, breast, and colonic origin, and were eliminated from the study. The remaining 43 were stratified by race and sex. There were 31 women (72\%) and 12 men (28\%). There were 15 black and 15 white women, but 7 white men and 3 black men. The other 3 patients were of other racial descents. Of the total cohort, 17 were smokers or had a history of smoking, and 23 were non-smokers. The smoking history of 3 patients was unknown.

The most common presenting symptom was pain, described in 28 of 43 patients (65\%). Sixteen of 43 patients presented with more than one complaint, but the initially recorded concern was identified and stratified for each patient (Table 1). Preoperative diagnosis was predominantly acute cholecystitis $(n=15)$ (Table 2$)$.

Clinicopathological values were examined for elevated

Table 1. Primary complaints in presentation of gallbladder cancer.

\begin{tabular}{cc}
\hline Primary presenting symptom & Number of patients \\
\hline Pain & 28 \\
Jaundice & 4 \\
Asymptomatic & 4 \\
Elective cholecystectomy & 2 \\
Weight loss & 2 \\
Nausea/vomiting & 2 \\
Change in bowel habits & 1 \\
\hline
\end{tabular}

Table 2. Preoperative diagnosis in presentation of gallbladder cancer (GBC).

\begin{tabular}{cc}
\hline Preoperative diagnosis & Number of cases \\
\hline Acute cholecystitis & 15 \\
GBC mass or previous GBC diagnosis & 9 \\
Cholelithiasis & 8 \\
Other cancer/mass & 4 \\
Chronic cholecystitis & 2 \\
Obstructive jaundice & 2 \\
Hydrops gallbladder & 1 \\
\hline
\end{tabular}


white blood cell (WBC) counts and total bilirubin (TB). Laboratory data were missing in one patient. Average WBC was 10.2 (range, 3.5 - 20.9). WBC (normal, 4.5 10.0) was elevated in 20 of $42(48 \%)$. Correlation with clinical presentation showed elevated WBC in 7 of 15 (47\%) patients suspected to have an inflammatory condition or presentation. Total bilirubin (normal, 0.3 - 1.9) was recorded in 36 patients, with 7 elevated (19\%). Eighteen patients were evaluated for tumor markers including CA19-9, CEA, CA125 and AFP. CA19-9 levels (range, 2 - 70,000; average 7279) were determined in 14 of the 18 patients; the levels were elevated (normal, <35 $\mathrm{U} / \mathrm{mL}$ ) in 9 patients (64\%). CEA, AFP, and CA125 were randomly elevated in a few patients but with no statistical significance or trends. From the 38 patients with available imaging studies, modalities most frequently used were US and CT (Table 3). Diagnosis of GBC was made either preoperatively, intraoperatively, or postoperatively from pathological analysis of tissue (Figure 1).

Of the 43 patients in this study, 5 pathology reports were unavailable. Of the remaining 38 patients, 17 had stage IV (12 were IVB), 6 had stage IIIA, 5 had stage II and 7 had stage I adenocarcinoma of the gallbladder. Two patients had carcinoma in situ (stage 0) and 1 had carcinoid of the gallbladder. Nodes were sent with pathological specimens in 19 patients, of which 9 patients had positive nodes (47\%) and 10 had benign nodes (53\%); 8 of the 9 patients with positive nodes were stage IVB. The positive nodes included pericystic, cystic duct, common bile duct, retroperitoneal, porta hepatis, retrocrural, pericholedochal, and paraduodenal. One patient had positive pericolic nodes that were more likely related to her diagnosed rectal cancer than her stage I GBC.

Cholecystectomies were planned initially in 26 of 43 cases (Figure 2). Radical or extended resections were only performed for 5 patients (12\%), and one of these was a port site resection for incidental stage II GBC found on laparoscopic cholecystectomy for benign disease. Further treatment included chemotherapy and radiation. In 16 of 43 cases, chemotherapy was undertaken
(38\%). There was no consistency with regards to the type of agent used, although gemcitabine was frequently employed. Radiation was applied in 4 cases (9\%).

Thirteen of 43 patients were lost to follow-up. Of the 30 patients deceased or confirmed living, the 5-year survival rate was $13 \%$. From the total 43 patients, there were 12 cases of early GBC (stages 1 \& 2) with an average survival of 55 months and a 5-year survival of $43 \%$. In 23 patients with late GBC (stages 3 \& 4), the average survival was 9 months with no survival at 5 years. The average and 5-year survivals were calculated from the 30 patients whose survival outcomes were known. Survival probability in early and late stage GBC is seen in Figure 3. Survival probability based on nodal status is seen in Figure 4. Those patients lost to follow up were factored into the Kaplan-Meier survival probability as the "number censored", based on the number of years from the time of diagnosis to the end of the study period.

\section{Discussion}

GBC is the most frequent biliary malignancy. It is rare, characterized by silent presentation, poor prognosis, and

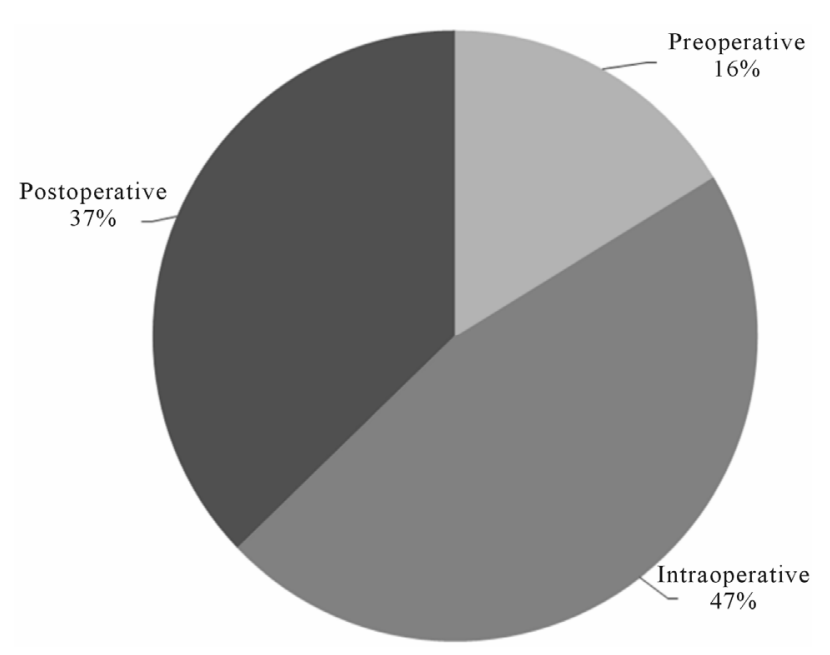

Figure 1. Time of diagnosis of GBC.

Table 3. Imaging studies in the diagnosis of gallbladder cancer (GBC).

\begin{tabular}{|c|c|c|}
\hline Type of imaging & Number of imaging studies completed & Number diagnosed with suspicious GBC mass \\
\hline Ultrasound & 25 & 4 \\
\hline Computed tomography & 22 & 5 \\
\hline HIDA scan & 9 & 0 \\
\hline Magnetic resonance imaging & 5 & 1 \\
\hline Endoscopic retrograde cholangiopancreatogram & 5 & 1 \\
\hline Magnetic resonance cholangiopancreatogram & 1 & 0 \\
\hline No studies available from charts & 7 & $\mathrm{n} / \mathrm{a}$ \\
\hline
\end{tabular}




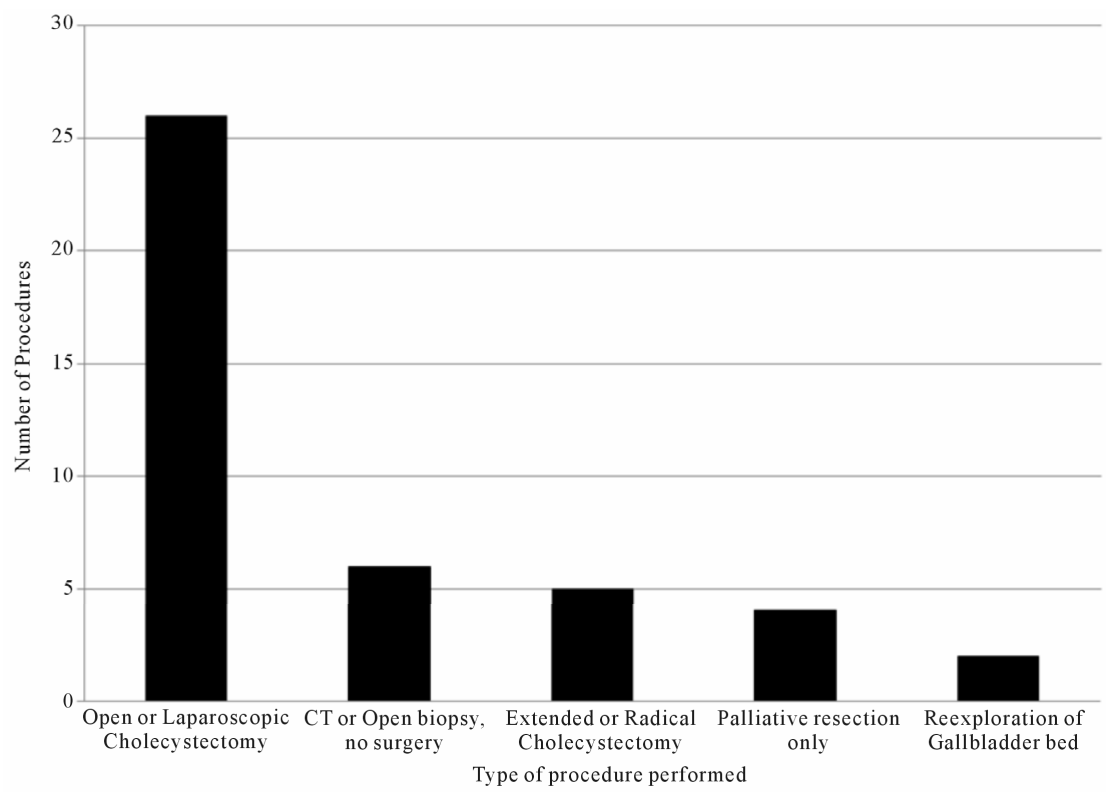

Figure 2. Type of procedure performed for GBC.

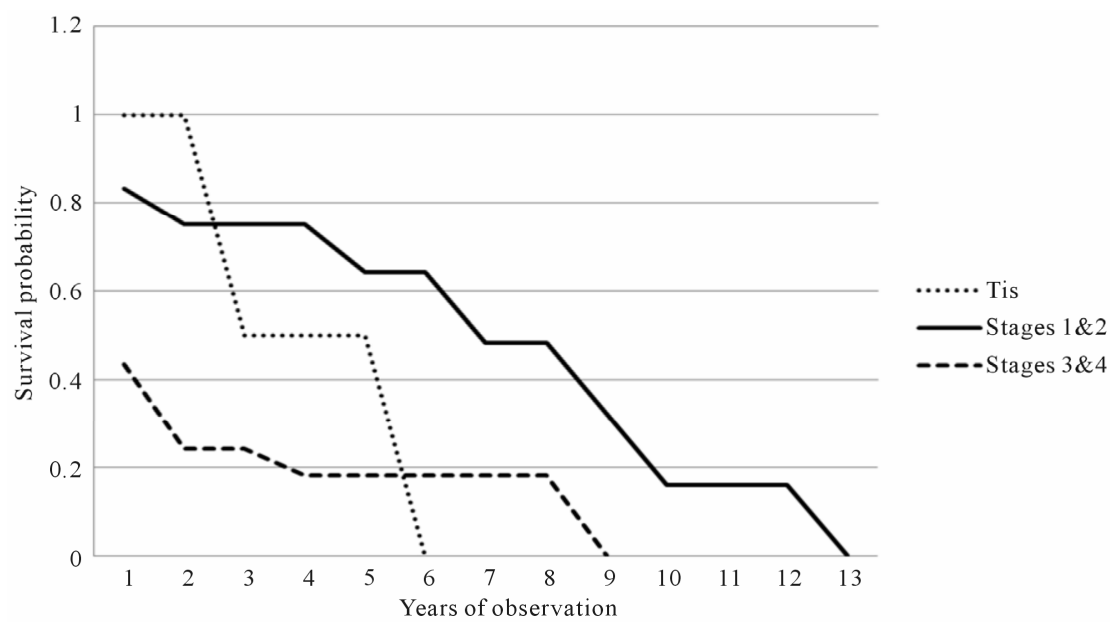

Figure 3. Kaplan-meier survival probability graph for carcinoma in situ, early (stages 1 \& 2), and late (stages 3 \& 4) GBC.

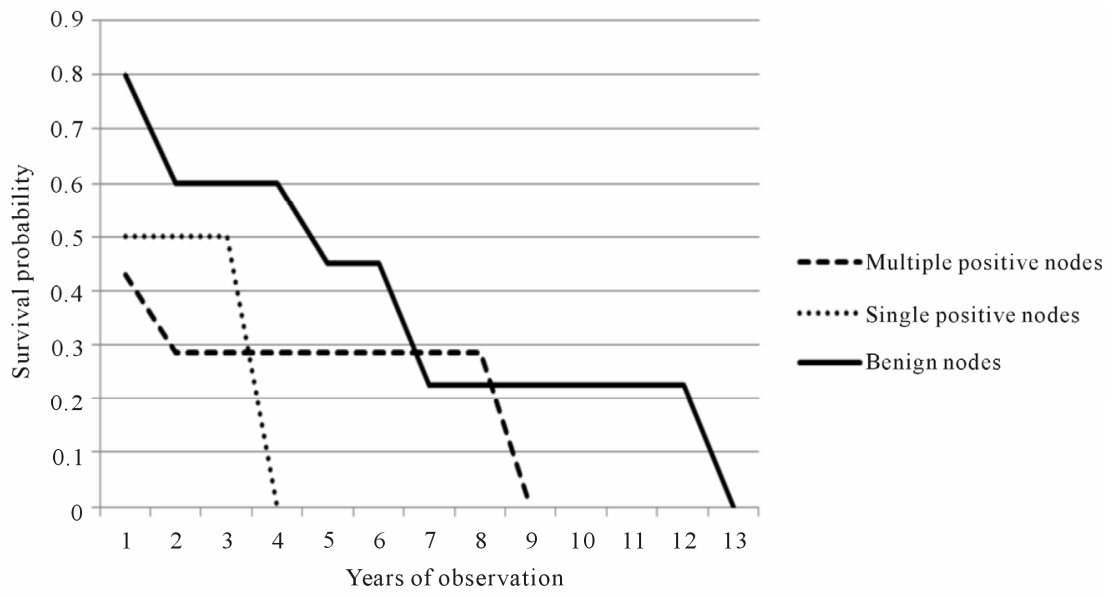

Figure 4. Kaplan-meier survival probability graph for known nodal status of patients with GBC. 
limited therapy [9]. Cholesterol gallstones, found in 35\% of women and $20 \%$ of men over age 75 , are known promoters of GBC and other biliary tract cancers $[1,3,4$, $10-12]$. GBC increases in frequency with age, and is correlated to the presence of gallstones in up to $94 \%$ of cases $[1,13]$. Although gallstones are frequently found in specimens with GBC, only $1 \%-3 \%$ of patients develop GBC after 10 - 25 years of asymptomatic cholelithiasis [1, $9,14]$. Our study had a mean age of 69 and 53\% had gallstones on imaging. GBC also has a propensity for the female sex, likely because of the increased gallstone disease seen in both obesity and pregnancy $[15,16]$. Obesity increases the risk of death from GBC, especially with a body mass index $>35$ [17]. The data from our study included more women than men, but BMI was not an available parameter. Smoking is another known risk factor for gallbladder cancer, but our study featured more non-smokers (58\%). This may have been a result of both small study size and retrospective approach. Other risk factors include constipation, chronic typhoid infection, chronic cholecystitis, an anomalous junction of pancreaticobiliary duct (AJPBD), and genetic predisposition $[3,4,10,18]$. Chronic cholecystitis was the admitting diagnosis in only $5 \%$ of patients in this study. No patients had a known history of chronic typhoid or AJPBD. Constipation was not a primary complaint.

Typically GBC is symptomatic only in advanced disease [19]. When symptoms occur, they are often related to coexisting cholelithiasis [20]. The most common complaints are pain or weight loss, although nausea has been noted at $40 \%-50 \%$ [21-24]. Pain was the presenting complaint in a majority of patients in this study. More often, GBC is incidentally discovered in a surgical specimen after resection for benign disease [2]. A majority of the patients in this study presented with symptoms and clinical markers, such as WBC and bilirubin, indistinguishable from gallstone disease. An elevated WBC count was often confounding in patients with GBC who present concurrently with acute cholecystitis or other acute or chronic inflammatory processes. Bilirubin is elevated in obstructive jaundice, but jaundice is described in the literature as a late finding in GBC; bilirubin is therefore not of great value in early diagnosis for preoperative management of early GBC [24]. Elevated bilirubin or jaundice also obscures the final diagnosis when imaging is unclear and pancreatic or ampullary masses are on the differential. One of the patients in this case series had an elevated bilirubin and concurrently had GBC and pancreatic cancer with elevated tumor markers.

Tumor markers are important in the preoperative workup of various gastrointestinal cancers. In GBC, tumor markers are related to the transformation and proliferation of neoplastic cells, but have yet to show prognostic value or survival benefit $[25,26]$. Most clinical studies only sporadically determine tumor markers in GBC, likely due to the lack of suspicion for the disease. Recent studies in tumor markers that show that CA15-3 expression can assist in discriminating between GBC and chronic cholelithiasis, but tumor markers in this study were limited to CEA, CA19-9, CA125, and AFP [25]. Elevated CA19-9 level has been described as an independent variable predictive of poor survival in GBC [27]. Another review held that CEA and CA19-9 levels were statistically insignificant in malignant polypoid lesions of the gallbladder, but that the markers were commonly drawn for assessing GBC [28]. CA19-9 is not specific to the diagnosis of GBC; it can also be elevated in other cancers and diseases of the bile ducts [29]. In this study, the advanced cancers (stages $3 \&$ 4) had more consistently elevated CA19-9 levels, but the range varied. The quantity of the marker did not correlate well with length of survival, although the population size was too small to draw any significant conclusions. The patient with the CA19-9 level of 70000 had multiple primary cancers. Of those patients with elevated CA19-9 levels, the average survival was 8.3 months. From observation alone, the results of this case series suggest that determining CA 19-9 levels preoperatively could be helpful, but not necessarily diagnostic of GBC or indicative of survival.

The pathogenesis of gallbladder carcinoma is unclear. One hypothesis involves the dysplasia-carcinoma sequence in the malignant transformation of epithelial cell cancers. This transformation has been similarly noted in ulcerative colitis, colon cancer, primary sclerosing cholangitis and cholangiocarcinoma [30]. Dysplastic tissue is often found next to carcinoma in situ in gallbladder specimens, suggesting that progression of dysplasia to carcinoma is a possible mechanism [10]. Overexpression of p53 in early GBC, frequently due to a missense mutation in exons 5,6 , or 8 , is increased in comparison with benign lesions or dysplasia; invasive GBC and high grade dysplasia also overexpress p53 [26,31,32]. In patients with chronic reflux and with gallstones $>3 \mathrm{~cm}$ in size, genetic mutations in p53 and K-ras have been noted $[3,10,14,33]$. In patients with AJPBD, there is a significant increase in mutations of codon 12 in the K-ras gene $[34,35]$. Recent studies suggest that increased expression of cell adhesion membrane proteins such as ADAM-17, which activate epidermal growth factor receptor (EGFR) and transforming growth factor alpha (TGF- $\alpha$ ), independently correlate to prognosis in GBC [36]. The genetic developments regarding the pathogenesis of GBC offers insights into new therapeutic and monitoring targets. Although some histological markers were identified in pathology reports of this study, there was no mention of K-ras or p53 in any case. It would be of value to remark on these markers of cellular dysplasia with regards to GBC, especially since the natural history of GBC is 
still under consideration, but there is limited preoperative value in these histological factors unless acquired under biopsy.

Despite improvements in imaging, post-operative pathology remains the main diagnostic method for GBC [30]. The results of this study are consistent with the literature. Studies suggest that US cannot distinguish between GBC and chronic cholecystitis and sensitivity remains low $[3,20]$, whereas others propose US is the best diagnostic method [22]. CT tends to have higher preoperative sensitivity, but depends on the morphology of the neoplasm $[3,37]$. Both US and CT are limited in the detection of small lesions [5], and US is not accurate in determining lymph node involvement or peritoneal dissemination [22]. GBC can present in two types of lesions: protruding and flat. Flat lesions are known for difficulty in diagnosis [7]. In one study, US detected intraluminal GBC in 59\% of cases, and infiltrative in $41 \%$ [22]. Wakai et al found suspicious lesions on US in $44 \%$ but could not determine the depth of invasion, which is important in the prognosis of early GBC [38]. Suspicious imaging for GBC includes polyps greater than $10 \mathrm{~mm}$, porcelain gallbladders, diffuse irregular thickening of the gallbladder wall, and mass filling or replacing defects [23]. Other forms of imaging enhance diagnosis. Endoscopic ultrasound (EUS) can detect early changes in the malignancy of polyps and endoscopic retrograde cholangiopancreatogram (ERCP) helps evaluate the extent of spread in GBC presenting with jaundice but typically EUS, ERCP, magnetic resonance angiography (MRA) and PET are not useful in identifying primary lesions $[2,3,13,33]$. Intraoperative US has a role in evaluating the extent of spread into the liver bed which dictates surgical course to obtain R0 resection [23]. The results of this study had a preoperative diagnosis of suspected GBC or mass in 4 of 25 available US (16\%) and 5 of 22 available CT scans (23\%), but no intraoperative US was used. Modifications in imaging techniques need to occur if imaging is to play a larger role in staging or diagnosis of early GBC [39].

Metastatically, GBC is peculiar as it spreads in various directions, often related to the position of the tumor [40]. Eighty-five to $90 \%$ of GBC are adenocarcinoma, with $60 \%$ found in the fundus [3]. GBC spreads by lymphatic, vascular, neural, intraperitoneal, intraductal and direct anatomical spread $[6,9,41]$. Direct invasion in the liver bed occurs in $34 \%$ - $89 \%$ of cases, and has been noted into the colon, bile duct, duodenum, and pancreas [19,42, 43]. Lymphatic drainage follows one of three routes: 1) right half of hepatoduodenal ligament runs into the cystic, pericholedochal and proper hepatic artery nodes then on to the superior retropancreaticoduodenal or retroportal nodes and finally the para-aortic nodes, 2) left reaches the retropancreaticoduodenal or retroportal nodes and goes medial to the hepatoduodenal ligament, and 3) to the he- patic hilus, which then spreads deep along Glissen's capsule $[6,19]$. Sentinel nodes are contentious, but the cystic node, pericholedochal node, retroportal node and hilar node are considered the first stations of lymphatic spread, and could be effective for sentinel lymph node biopsy [40]. In one study, only nodal involvement was an independent prognostic factor on multivariate analysis [13]. Five- and ten-year survival decreases when the number of involved nodes exceeds one [40]. In this study, there was a decrease in survival with node-positive status. Early GBC patients in this study had a 1-year survival of $71 \%$, but this decreased to $50 \%$ with a single positive node and $20 \%$ with multiple positive nodes. No nodepositive patients survived to 5 years. Survival with nodes around the pancreas and celiac artery (N2 status by TNM staging) is better if there are no metastases and no nodal involvement of the para-aortic nodes [8]. There is still no conclusion on the degree of lymph node dissection resulting in the best prognosis for GBC, although radical dissection with $\mathrm{R} 0$ resection in $\mathrm{T} 2$ or greater tumors may be beneficial $[43,44]$. Only 5 extended or radical procedures were performed in this study with no statistical benefit to survival.

Surgical approach is dictated by the stage of GBC. Carcinoma in situ and T1a GBC are cured by simple cholecystectomy, but efficacy is limited if the cancer invades the serosa [41]. T1b or T2 cancers require a wedge resection, or extended cholecystectomy, for improved survival. This demands the resection of $2 \mathrm{~cm}$ of liver tissue from the gallbladder bed and regional lymphadenectomy $[3,7,41,45]$. Laparoscopic cholecystectomies must be converted to open if GBC is found intraoperatively as seeding occurs in up to $17 \%$ [6]. Advanced T2/T3 and/or N1 cancer require radical cholecystectomies. This includes en-bloc subsegmental resection of hepatic segments IVB and V and regional lymphadenectomy of hepatoduodenal ligament, common hepatic artery, and retropancreatic nodes $[5,46,47]$. A trisectionectomy with bile duct resection can be used if the infundibulum, gallbladder neck, or triangle of Calot is involved [6,47], although some studies report higher post-operative hepatic failure and recurrence, even with R0 resection [24,48]. These procedures, however, carry significant morbidity and often multiple node cancers have too high a recurrence risk $[2,40]$. One study did show an increase of 5-year survival for advanced GBC with positive peripancreatic nodes and no distant metastases after combined pancreaticoduodenectomy and hepatectomy, but others show minimal change of 5-year survival $[42,47,48]$. Advances in radical surgery altered the surgical approach to GBC in the 1990s and has been improving survival [47]. In our study, 5 surgeries were radical resections. One patient was lost to follow-up, but the range of survival for the other 4 patients was 2 weeks to 51 months, with an 
average survival of 21 months.

Adjuvant therapy for GBC is still under debate $[43,49]$. Radiation therapy only shows a survival benefit in cases with microscopic positive margins or residual disease after resection $[6,20,46]$. Radiation therapy was used in $9 \%$ of the patients in this study for palliation only. Intraoperative radiation has been used for local residual disease with limited benefit [46]. No intraoperative radiation was used in this study. Some survival benefit has been shown combining 5-fluorouracil (5-FU) and external beam radiation over patients who only received surgery [6]. Gemcitabine has shown varied responses, from none to equivalence with 5-FU and leukovorin; 3-AP, capecitabine, oxaliplatin and bevacizumab are being tested $[3,40,43,49,50]$. Mitomycin C (MMC) and 5-FU for isolated para-aortic node recurrence had overall improvements in 5-year survival [51,52]. High-dose 5-FU shows partial response in biliary tract cancer, with or without additional agents, likely due to the increased effect of 5-FU on p53-expressing tissue [52,53]. In this study, $37 \%$ of patients had chemotherapy using various regimes, including gemcitabine and 5-FU, but there was no survival benefit. More investigation into the type, quantity and timing of adjuvant therapy is needed in the treatment of GBC.

Average survival has been noted as 36 months, although this varies with grade and stage of cancer [30]. The average survival in this study was 28 months, with the overall 5-year survival at $13 \%$. The 5-year survival for the advanced GBC (stages III and IV) in this study was $0 \%$, with an average survival of 9 months. Recurrence is found mainly in systemic lymph nodes, remnant liver, peritoneum secondary to local invasion, nodal spread, seeding, or distant metastases [13,40,54]. Surgical exploration for possible recurrence occurred in 2 patients who had been previously resected at other facilities. $\mathrm{R} 0$ resection from the initial operation remains the best method of preventing early local recurrence and achieving long term survival $[9,47,48]$.

The limitations of our study are common for retrospective studies conducted over a significant period of time. A small sample size was expected due to the rarity of GBC, but still affects the results. There was a change in the management of GBC in western practice in the 1990s, which resulted in changes not only in the type of operations performed, but also in imaging, data reporting, and operating personnel. Bias must definitely be considered regarding average survival time and 5-year survival rates secondary to the number of patients lost to follow up, although these patients were factored into the survival probability graphs. Regardless of these potential sources of bias, the trends of this paper underline the malignant nature of a disease that requires high levels of clinical suspicion and improvements in preoperative diagnosis.
This is consistent with, and adds to, the literature on GBC.

\section{Conclusion}

Simple cholecystectomy for early GBC, and radical resection (determined by the grade, nodal status, and direction of spread) for cancers T1b or greater, are the surgical options for successful resection. There is still considerable overlap between the clinical presentation of GBC and more benign inflammatory conditions such as acute cholecystitis when laboratory values and clinical parameters are used for diagnosis. Improvements in imaging and preoperative planning are mandatory. Investigations into the use of tumor markers (e.g., CA15-3) and genetics (e.g., K-ras and p53) will become the key elements to improving outcomes in this insidious, lethal disease. Survival rates for advanced disease and nodepositive status can be improved by early diagnosis and curative resection.

\section{REFERENCES}

[1] D. F. Ransohoff and W. A. Gracie, "Treatment of Gallstones,” Annals of Internal Medicine, Vol. 119, No. 7, 1993, pp. 606-619.

[2] C. D. Anderson, M. H. Rice, C. W. Pinson, et al., "Fluorodeoxyglucose PET Imaging in the Evaluation of Gallbladder Carcinoma and Cholangiocarcinoma," Journal of Gastrointestinal Surgery, Vol. 8, No. 1, 2004, pp. 90-97. doi:10.1016/j.gassur.2003.10.003

[3] K. M. Reid, M. A. de la Ramos and J. H. Donohue, "Diagnosis and Surgical Management of Gallbladder Cancer: A Review,” Journal of Gastrointestinal Surgery, Vol. 11, No. 5, 2007, pp. 671-681. doi:10.1007/s11605-006-0075-X

[4] G. Randi, S. Franceschi and V. C. La, "Gallbladder Cancer Worldwide: Geographical Distribution and Risk Factors," International Journal of Cancer, Vol. 118, No. 7, 2006, pp. 1591-1602. doi:10.1002/ijc.21683

[5] X. de Aretxabala, I Roa, L. Burgos, et al., "Gallbladder Cancer: An Analysis of a Series of 139 Patients with Invasion Restricted to the Subserosal Layer," Journal of Gastrointestinal Surgery, Vol. 10, No. 2, 2006, pp. 186192. doi:10.1016/j.gassur.2005.11.003

[6] K. L. Mekeel and A. W. Hemming, "Surgical Management of Gallbladder Carcinoma: A Review,” Journal of Gastrointestinal Surgery, Vol. 11, No. 9, 2007, pp. 11881193. doi:10.1007/s11605-007-0115-1

[7] H. Onoyama, M. Yamamoto, M. Takada, et al., "Diagnostic Imaging of Early Gallbladder Cancer: Retrospective Study of 53 Cases," World Journal of Surgery, Vol. 23, No. 7, 1999, pp. 708-712. doi:10.1007/PL00012373

[8] S. Kondo, Y. Nimura, N. Hayakawa, et al., "Regional and Para-Aortic Lymphadenectomy in Radical Surgery for Advanced Gallbladder Carcinoma," British Journal of Surgery, Vol. 87, No. 4, 2000, pp. 418-422. 
doi:10.1046/j.1365-2168.2000.01384.X

[9] M. C. Misra and S. Guleri, "Management of Cancer Gallbladder Found as a Surprise on a Resected Gallbladder Specimen,” Journal of Surgical Oncology, Vol. 93, No. 8, 2006, pp. 690-698. doi:10.1002/jso.20537

[10] E. C. Lazcano-Ponce, J. F. Miquel, N. Munoz, et al., "Epidemiology and Molecular Pathology of Gallbladder Cancer," CA: A Cancer Journal for Clinicians, Vol. 51, No. 6, 2001, pp. 349-364. doi:10.3322/canjclin.51.6.349

[11] F. Levi, F. Lucchini, E. Negri and V. C. La, "The Recent Decline in Gallbladder Cancer Mortality in Europe," European Journal of Cancer Prevention, Vol. 12, No. 4, 2003, pp. 265-267. doi:10.1097/00008469-200308000-00004

[12] W. H. Chow, C. Johansen, G. Gridley, et al., "Gallstones, Cholecystectomy and Risk of Cancers of the Liver, Biliary Tract and Pancreas," British Journal of Cancer, Vol. 79, 1999, pp. 640-644. doi:10.1038/sj.bjc.6690101

[13] D. L. Bartlett, Y. Fong, J. G. Fortner, et al., "Long-Term Results after Resection for Gallbladder Cancer. Implications for Staging and Management," Annals of Surgery, Vol. 224, No. 5, 1996, pp. 639-646. doi:10.1097/00000658-199611000-00008

[14] A. K. Diehl, "Gallstone Size and the Risk of Gallbladder Cancer," Journal of the American Medical Association, Vol. 250, No. 17, 1983, pp. 2323-2326. doi:10.1001/jama.1983.03340170049027

[15] C. J. Moerman, M. P. Berns, H. B. Bueno de Mesquita and S. Runia, "Reproductive History and Cancer of the Biliary Tract in Women," International Journal of Cancer, Vol. 57, No. 2, 1994, pp. 146-153. doi:10.1002/ijc.2910570203

[16] M. Pandey and V. K. Shukla, "Lifestyle, Parity, Menstrual and Reproductive Factors and Risk of Gallbladder Cancer," European Journal of Cancer Prevention, Vol. 12, No. 4, 2003, pp. 269-272. doi:10.1097/00008469-200308000-00005

[17] E. E. Calle, C. Rodriguez, K. Walker-Thurmond and M. J. Thun, "Overweight, Obesity, and Mortality from Cancer in a Prospectively Studied Cohort of U.S. Adults," The New England Journal of Medicine, Vol. 348, 2003, pp. 1625-1638. doi:10.1056/NEJMoa021423

[18] K. Yagyu, Y. Lin, Y Obata, et al., "Bowel Movement Frequency, Medical History and the Risk of Gallbladder Cancer Death: A Cohort Study in Japan,” Cancer Science, Vol. 95, No. 8, 2004, pp. 674-678. doi:10.1111/j.1349-7006.2004.tb03328.x

[19] A. Muratore, R. Polastri and L. Capussotti, "Radical Surgery for Gallbladder Cancer: Current Options,” European Journal of Surgical Oncology, Vol. 26, No. 5, 2000, pp. 438-443. doi:10.1053/ejso.1999.0918

[20] J. H. Donohue, A. K. Stewart and H. R. Menck, "The National Cancer Data Base Report on Carcinoma of the Gallbladder, 1989-1995,” Cancer, Vol. 83, No. 12, 1998, pp. 2618-2628.

doi:10.1002/(SICI)1097-0142(19981215)83:12<2618::AI D-CNCR29>3.0.CO;2-H
[21] Y. Fong, W. Jarnagin and L. H. Blumgart, “Gallbladder Cancer: Comparison of Patients Presenting Initially for Definitive Operation with Those Presenting after Prior Noncurative Intervention,” Annals of Surgery, Vol. 232, No. 4, 2000, pp. 557-569. doi:10.1097/00000658-200010000-00011

[22] M. Pandey, B. P. Sood, R. C. Shukla, et al., "Carcinoma of the Gallbladder: Role of Sonography in Diagnosis and Staging,” Journal of Clinical Ultrasound, Vol. 28, No. 5, 2000, pp. 227-232. doi:10.1002/(SICI)1097-0096(200006)28:5<227::AID-JC $\underline{\mathrm{U} 4>3.0 . \mathrm{CO} ; 2-4}$

[23] J. C. Box and S. B. Edge, "Laparoscopic Cholecystectomy and Unsuspected Gallbladder Carcinoma," Seminars in Surgical Oncology, Vol. 16, 1999, pp. 327-331. doi:10.1002/(SICI)1098-2388(199906)16:4<327::AID-SS $\underline{\mathrm{U} 8>3.0 . \mathrm{CO} ; 2-\mathrm{V}}$

[24] W. G. Hawkins, R. P. DeMatteo, W. R. Jarnagin WR, et al., "Jaundice Predicts Advanced Disease and Early Mortality in Patients with Gallbladder Cancer," Annals of Surgical Oncology, Vol. 11, 2004, pp. 310-315. doi:10.1245/ASO.2004.03.011

[25] M. Artico, E. Bronzetti, V. Alicino, et al., "Human Gallbladder Carcinoma: Role of Neurotrophins, MIB-1, CD34 and CA15-3," European Journal of Histochemistry, Vol. 54, 2010, p. e10. doi:10.4081/ejh.2010.e10

[26] K. Fujii, H. Yokozaki, W. Yasui, et al., "High Frequency of p53 Gene Mutation in Adenocarcinomas of the Gallbladder," Cancer Epidemiology, Biomarkers \& Prevention, Vol. 5, 1996, pp. 461-466.

[27] I. Hatzaras, C. Schmidt, P. Muscarella, et al., "Elevated CA 19-9 Portends Poor Prognosis in Patients Undergoing Resection of Biliary Malignancies,” HPB (Oxford), Vol. 12, No. 2, 2010, pp. 134-138. doi:10.1111/j.1477-2574.2009.00149.x

[28] W. Kwon, J. Y. Jang, S. E. Lee, et al., "Clinicopathologic Features of Polypoid Lesions of the Gallbladder and Risk Factors of Gallbladder Cancer," Journal of Korean Medical Science, Vol. 24, No. 3, 2009, pp. 481-487. doi:10.3346/jkms.2009.24.3.481

[29] A. H. Patel, D. M. Harnois, G. G. Klee, et al., "The Utility of CA 19-9 in the Diagnoses of Cholangiocarcinoma in Patients without Primary Sclerosing Cholangitis," American Journal of Gastroenterology, Vol. 95, No. 1, 2000, pp. 204-207. doi:10.1111/j.1572-0241.2000.01685.x

[30] D. C. Buckles, K. D. Lindor, N. F. Larusso, et al., "In Primary Sclerosing Cholangitis, Gallbladder Polyps Are Frequently Malignant," American Journal of Gastroenterology, Vol. 97, 2002, pp. 1138-1142. doi:10.1111/j.1572-0241.2002.05677.x

[31] T. Itoi, H. Watanabe, M. Yoshida, et al., "Correlation of p53 Protein Expression with Gene Mutation in GallBladder Carcinomas,” Pathology International, Vol. 47, 1997, pp. 525-530. doi:10.1111/j.1440-1827.1997.tb04535.x

[32] A. Wee, M. Teh and G. C. Raju, “Clinical Importance of p53 Protein in Gall Bladder Carcinoma and Its Precursor 
Lesions,” Journal of Clinical Pathology, Vol. 47, 1994, pp. 453-456. doi:10.1136/jcp.47.5.453

[33] S. Tazuma and G. Kajiyama, "Carcinogenesis of Malignant Lesions of the Gall Bladder. The Impact of Chronic Inflammation and Gallstones," Langenbeck's Archives of Surgery, Vol. 386, No. 3, 2001, pp. 224-229. doi:10.1007/s004230100220

[34] M. Tada, O. Yokosuka, M. Omata, et al., "Analysis of Ras Gene Mutations in Biliary and Pancreatic Tumors by Polymerase Chain Reaction and Direct Sequencing," Cancer, Vol. 66, 1990, pp. 930-935. doi:10.1002/1097-0142(19900901)66:5<930::AID-CNCR 2820660519>3.0.CO;2-W

[35] K. Hanada, M. Itoh, K. Fujii, et al., "K-ras and p53 Mutations in Stage I Gallbladder Carcinoma with an Anomalous Junction of the Pancreaticobiliary Duct," Cancer, Vol. 77, 1996, pp. 452-458. doi:10.1002/(SICI)1097-0142(19960201)77:3<452::AIDCNCR5>3.0.CO;2-M

[36] K. Wu, M. Liao, B. Liu and Z. Deng, “ADAM-17 OverExpression in Gallbladder Carcinoma Correlates with Poor Prognosis of Patients,” Medical Oncology, Vol. 28, No. 2, 2011, pp. 475-480. doi:10.1007/s12032-010-9481-8

[37] T. Y. Lee, S. F. Ko, C. C. Huang, et al., "Intraluminal versus Infiltrating Gallbladder Carcinoma: Clinical Presentation, Ultrasound and Computed Tomography," World Journal of Gastroenterology, Vol. 15, No. 45, 2009, pp. 5662-5668. doi:10.3748/wjg.15.5662

[38] T. Wakai, Y. Shirai, N. Yokoyama, et al., "Early Gallbladder Carcinoma Does Not Warrant Radical Resection,” British Journal of Surgery, Vol. 88, No. 5, 2001, pp. 675678. doi:10.1046/j.1365-2168.2001.01749.x

[39] Y. Fong, L. Wagman, M. Gonen, et al., "Evidence-Based Gallbladder Cancer Staging: Changing Cancer Staging by Analysis of Data from the National Cancer Database,” Annals of Surgery, Vol. 243, No. 6, 2006, pp. 767-771. doi:10.1097/01.sla.0000219737.81943.4e

[40] I. Endo, H. Shimada, M. Tanabe, et al., "Prognostic Significance of the Number of Positive Lymph Nodes in Gallbladder Cancer,” Journal of Gastrointestinal Surgery, Vol. 10, No. 7, 2006, pp. 999-1007. doi:10.1016/j.gassur.2006.03.006

[41] H. Onoyama, M. Yamamoto, A. Tseng, et al., "Extended Cholecystectomy for Carcinoma of the Gallbladder," World Journal of Surgery, Vol. 19, No. 5, 1995, pp. 758763. doi:10.1007/BF00295925

[42] Y. Ogura, R. Mizumoto, S. Isaji, et al., "Radical Operations for Carcinoma of the Gallbladder: Present Status in Japan,” World Journal of Surgery, Vol. 15, 1991, pp. 337343. doi:10.1007/BF01658725

[43] H. A. Pitt, "Gallbladder Cancer: What Is an Aggressive Approach?” Annals of Surgery, Vol. 241, No. 3, 2005, pp. 395-396. doi:10.1097/01.sla.0000154119.55201.e9

[44] Y. Shirai, T. Wakai and K. Hatakeyama, "Radical Lymph Node Dissection for Gallbladder Cancer: Indications and Limitations," Surgical Oncology Clinics of North Ameri ca, Vol. 16, 2007, pp. 221-232.

\section{doi:10.1016/j.soc.2006.10.011}

[45] K. Yamaguchi, K. Chijiiwa, S. Saiki, et al., "Retrospective Analysis of 70 Operations for Gallbladder Carcinoma,” British Journal of Surgery, Vol. 84, No. 2, 1997, pp. 200-204. doi:10.1002/bjs.1800840217

[46] T. Todoroki, T. Kawamoto, H. Takahashi, et al., "Treatment of Gallbladder Cancer by Radical Resection,” British Journal of Surgery, Vol. 86, No. 5, 1999, pp. 622-627. doi:10.1046/j.1365-2168.1999.01085.X

[47] E. Dixon, C. M. Vollmer Jr., A. Sahajpal, et al., “An Aggressive Surgical Approach Leads to Improved Survival in Patients with Gallbladder Cancer: A 12-Year Study at a North American Center," Annals of Surgery, Vol. 241, No. 3, 2005, pp. 385-394. doi:10.1097/01.sla.0000154118.07704.ef

[48] Y. Shirai, T. Ohtani, K. Tsukada and K. Hatakeyama, "Combined Pancreaticoduodenectomy and Hepatectomy for Patients with Locally Advanced Gallbladder Carcinoma: Long Term Results," Cancer, Vol. 80, 1997, pp. 1904-1909. doi:10.1002/(SICI)1097-0142(19971115)80:10<1904::AI D-CNCR5>3.3.CO;2-Z

[49] X. de Aretxabala, I. Roa, M. Berrios, et al., "Chemoradiotherapy in Gallbladder Cancer,” Journal of Surgical Oncology, Vol. 93, No. 8, 2006, pp. 699-704. doi:10.1002/jso.20538

[50] T. Andre, J. M. Reyes-Vidal, L. Fartoux, et al., "Gemcitabine and Oxaliplatin in Advanced Biliary Tract Carcinoma: A Phase II Study,” British Journal of Cancer, Vol. 99, 2008, pp. 862-867. doi:10.1038/sj.bjc.6604628

[51] S. R. Alberts, H. Al-Khatib, M. R. Mahoney, et al., "Gemcitabine, 5-Fluorouracil, and Leucovorin in Advanced Biliary Tract and Gallbladder Carcinoma: A North Central Cancer Treatment Group Phase II Trial,” Cancer, Vol. 103, No. 1, 2005, pp. 111-118. doi:10.1002/cncr.20753

[52] T. Takada, H. Amano, H. Yasuda, et al., "Is Postoperative adjuvant Chemotherapy Useful for Gallbladder Carcinoma? A Phase III Multicenter Prospective Randomized Controlled Trial in Patients with Resected Pancreaticobiliary Carcinoma," Cancer, Vol. 95, No. 8, 2002, pp. 1685-1695. doi:10.1002/cncr.10831

[53] V. Gebbia, E. Majello, A. Testa, et al., "Treatment of Advanced Adenocarcinomas of the Exocrine Pancreas and the Gallbladder with 5-Fluorouracil, High Dose Levofolinic Acid and Oral Hydroxyurea on a Weekly Schedule. Results of a Multicenter Study of the Southern Italy Oncology Group (G.O.I.M.),” Cancer, Vol. 78, 1996, pp. 1300-1307.

doi:10.1002/(SICI)1097-0142(19960915)78:6<1300::AID -CNCR19>3.3.CO;2-Y

[54] J. S. Park, D. S. Yoon, K. S. Kim, et al., "Actual Recurrence Patterns and Risk Factors Influencing Recurrence after Curative Resection with Stage II Gallbladder Carcinoma," Journal of Gastrointestinal Surgery, Vol. 11, No. 5, 2007, pp. 631-637. doi:10.1007/s11605-007-0109-Z 